\title{
Critical Temperature of a Trapped Bose Gas: Mean-Field Theory and Fluctuations
}

\author{
M. Houbiers, ${ }^{1}$ H.T.C. Stoof, ${ }^{1}$ and E.A. Cornell ${ }^{2}$ \\ ${ }^{1}$ Institute for Theoretical Physics, University of Utrecht, Princetonplein 5, \\ P.O. Box 80.006, 3508 TA Utrecht, The Netherlands \\ ${ }^{2}$ JILA, National Institute of Standards and Technology, University of Colorado \\ and \\ Physics Department, University of Colorado, Boulder CO 80309-0440
}

\begin{abstract}
We investigate the possibilities of distinguishing the mean-field and fluctuation effects on the critical temperature of a trapped Bose gas with repulsive interatomic interactions. Since in a direct measurement of the critical temperature as a function of the number of trapped atoms these effects are small compared to the ideal gas results, we propose to observe Bose-Einstein condensation by adiabatically ramping down the trapping frequency. Moreover, analyzing this adiabatic cooling scheme, we show that fluctuation effects can lead to the formation of a Bose condensate at frequencies which are much larger than those predicted by the mean-field theory.
\end{abstract}

PACS number(s): 03.75.Fi, 67.40.-w, 32.80.Pj, 42.50.Vk

The experimental observation of Bose-Einstein condensation in atomic gases of ${ }^{87} \mathrm{Rb}$ [1], ${ }^{23} \mathrm{Na}$ [2], and ${ }^{7} \mathrm{Li}$ [3] confined in a magnetic trap, has enormously boosted the theoretical and experimental research of such quantum gases. After studying the elementary excitations of the condensate [4,5], the JILA and MIT groups have also started to determine more accurately the critical temperature of the gas as a function of the number of atoms in the trap, and in particular the deviation of this temperature from the ideal case [6]. Measuring these deviations will give important information on the effect of interactions on the thermodynamic properties of the gas and may also signal a break-down of the Bogoliubov theory that has been so succesful in explaining the collective excitation spectrum [7 10]. It is therefore of considerable theoretical interest.

For a non-interacting gas in an isotropic external harmonic potential $V(\mathbf{r})=m \omega^{2} \mathbf{r}^{2} / 2$, it is well known that if the number of particles $N \gg 100$, Bose-Einstein condensation occurs at a temperature

$$
T_{0}=\left(\frac{N}{\zeta(3)}\right)^{1 / 3} \frac{\hbar \omega}{k_{B}}
$$

However, interactions between the atoms influence the critical behavior of the alkali gases of interest and shift the critical temperature. Roughly speaking there are two effects to consider. First, the inhomogeneity of the gas leads (above the transition) to a spatially-varying meanfield potential of $2 n(\mathbf{r}) T^{2 B}$, where $n(\mathbf{r})$ is the local density of the gas, $T^{2 B}=4 \pi a \hbar^{2} / m$ is the two-body transition matrix at zero energy and momenta, and $a$ is the $s$-wave scattering length. Depending on the sign of the scattering length the atoms are therefore either repelled from $(a>0)$ or attracted to $(a<0)$ the center of the trap and it requires more or less atoms respectively, to acquire the necessary central density for Bose-Einstein condensation. Using a local-density approximation this effect was recently studied by Giorgini, Pitaevskii and
Stringari [11]. They obtained a mean-field shift in the critical temperature of

$$
\frac{T_{c}-T_{0}}{T_{0}} \simeq-1.33 \frac{a}{l} N^{1 / 6}
$$

where $l=\sqrt{\hbar / m \omega}$ is the extent of the harmonic oscillator ground state. Secondly, Bijlsma and Stoof have found by means of a renormalization group calculation that for gases with a positive scattering length the necessary central density for Bose-Einstein condensation is reduced considerably due to fluctuation effects [12]. This means that the degeneracy parameter $n(\mathbf{0}) \Lambda_{t h}^{3}$ (with $\Lambda_{t h}=\left(2 \pi \hbar^{2} / m k_{B} T\right)^{1 / 2}$ the thermal de Broglie wavelength) at the critical temperature is not $\zeta(3 / 2) \simeq 2.612$, as assumed by the mean-field theory, but instead a monotonically decreasing function of the quantum parameter $a / \Lambda_{t h}$. It is therefore always smaller than the ideal gas value $\zeta(3 / 2)$.

Although the latter effect clearly increases the critical temperature, it is at present unclear to what amount this increase cancels the mean-field shift in the critical temperature. To investigate this issue, we need quantitative information on the density profile of a trapped Bose gas with repulsive interactions, which we obtain by performing numerical calculations in the local-density approximation. In this approximation the system is considered to be locally homogeneous, and the inhomogeneity induced by the trapping potential $V(\mathbf{r})$ and the meanfield interaction $2 n(\mathbf{r}) T^{2 B}$ is determined selfconsistently by the spatially-dependent effective chemical potential $\mu^{\prime}(\mathbf{r})=\mu-V(\mathbf{r})-2 n(\mathbf{r}) T^{2 B}$, where $\mu$ is the actual chemical potential of the gas.

For our purposes, which will become clear shortly, we also need to calculate the total number of particles $N$ in the gas, together with the entropy $S$. According to statistical physics, these quantities can be calculated from the grand-canonical potential $\Omega$ of the system, i.e. from 


$$
N=-\left.\frac{\partial \Omega}{\partial \mu}\right|_{T, V} \text { and } S=-\left.\frac{\partial \Omega}{\partial T}\right|_{\mu, V} .
$$

Above the critical temperature the (mean-field) grandcanonical potential of an interacting Bose gas is given by

$$
\begin{aligned}
\Omega=k_{B} T \sum_{j} \ln (1-\exp [ & \left.\left.-\beta\left(\epsilon_{j}-\mu\right)\right]\right) \\
& -\int d \mathbf{r} n^{2}(\mathbf{r}) T^{2 B},
\end{aligned}
$$

where $\epsilon_{j}$ denote the one-particle energy levels in the effective potential $V(\mathbf{r})+2 n(\mathbf{r}) T^{2 B}$ [13]. Implementing the local-density approximation by putting $\epsilon_{j}=\hbar^{2} \mathbf{k}^{2} / 2 m+$ $V(\mathbf{r})+2 n(\mathbf{r}) T^{2 B}$ and using $\sum_{j}=\int d \mathbf{r} \int d \mathbf{k} /(2 \pi)^{3}$, we can perform the gaussian integral over the momenta $\hbar \mathbf{k}$ to find

$$
-\frac{k_{B} T}{\Lambda_{t h}^{3}} \int d \mathbf{r} g_{5 / 2}[z(\mathbf{r})]
$$

for the first term on the right-hand side of Eq. (3). Here we introduced the Bose function

$$
g_{5 / 2}(z)=-\frac{4}{\sqrt{\pi}} \int_{0}^{\infty} d x x^{2} \ln \left(1-z \exp \left[-x^{2}\right]\right)
$$

and the local fugacity $z(\mathbf{r})=\exp \left[\beta \mu^{\prime}(\mathbf{r})\right]$.

Calculating now the derivative of $\Omega$ with respect to $\mu$, one obtains for the total number of particles

$$
N=\int d \mathbf{r} n(\mathbf{r})=\frac{1}{\Lambda_{t h}^{3}} \int d \mathbf{r} g_{3 / 2}[z(\mathbf{r})]
$$

where we used $g_{3 / 2}(z)=z\left[d g_{5 / 2}(z) / d z\right]$. Performing a similar differentiation with respect to the temperature, the entropy of the system is found to be

$$
S=k_{B} \int d \mathbf{r}\left\{\frac{5}{2} g_{5 / 2}[z(\mathbf{r})]-n(\mathbf{r}) \ln [z(\mathbf{r})]\right\} .
$$

Note that in both differentiations we used the fact that $z(\mathbf{r})$ has an implicit dependence on the chemical potential and the temperature through the density $n(\mathbf{r})$. The contributions to the particle number and the entropy from this dependence exactly cancel, however, the contributions coming from the second term in the right-hand side of Eq. (3).

At this point we are ready to discuss the way in which we numerically determine the critical temperature of the gas as a function of the number of particles in the trap and, in particular, how we include the fluctuation effects on this critical temperature. To include these fluctuations in a first approximation, we calculate at a given temperature the value of the quantum parameter $a / \Lambda_{t h}$ and extract from the appropriate value of the critical degeneracy parameter [12] the value of the central density $n(\mathbf{0})$ at which Bose-Einstein condensation first occurs. Knowing the critical central density we can also immediately determine the chemical potential at criticality from $n(\mathbf{0}) \Lambda_{t h}^{3}=g_{3 / 2}\left[\exp \left\{\beta\left(\mu-2 n(\mathbf{0}) T^{2 B}\right)\right\}\right]$. The corresponding number of particles is then self-consistently calculated from Eq. (5). To compare with the mean-field results, we also calculate the number of particles in the system for a central density of $\zeta(3 / 2) / \Lambda_{t h}^{3}$. Both results, together with Eq. (2) are plotted in Fig. 1.

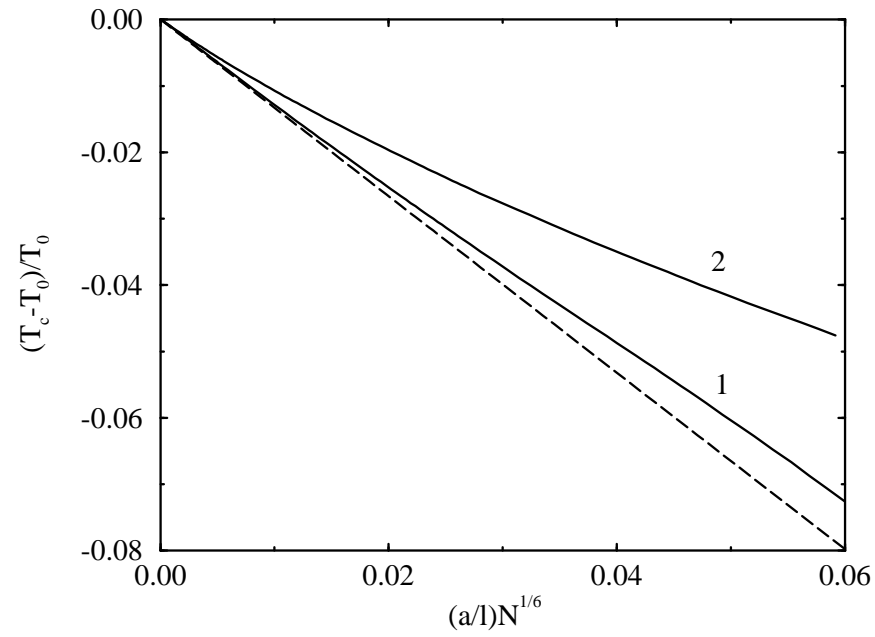

FIG. 1. Relative change in the critical temperature for an interacting gas in a harmonic oscillator trap. Curve 1 gives the mean-field result and curve 2 includes also the effect of fluctuations. The dashed line represents Eq. (2).

For different values of the scattering length $a$ and the trapping frequency $\omega$, we determined the critical temperature as a function of the number of particles and found that for both calculations the relative shift in the critical temperature is a universal function of $(a / l) N^{1 / 6}$ as indicated in Fig. 1. From this figure we see that Eq. (2) gives the correct lowest order mean-field shift of the temperature, but also that higher order corrections become important relatively soon. Moreover, the fluctuation effects are roughly speaking of the same order of magnitude under the experimental conditions of interest, although they are clearly unable to overcome the decrease of the critical temperature due to the mean-field potential. However, at this point we have to remind ourselves that the present calculation underestimates the influence of fluctuations because it does not incorporate the effect that fluctuations have on the density profile in the trap. In principle, this could also be included by performing what one might call a 'local renormalization group' calculation, i.e. apply the renormalization group theory of Ref. [12] at each point in space.

Since the critical temperature of an interacting Bose gas in an absolute sense does not deviate much from the ideal gas value, we now propose a possible experiment that could make the effects of the interatomic interactions visible without having to deal with a large ideal gas 'background'. Suppose we have a gas of $N$ particles in an isotropic trap with frequency $\omega$ and at a temperature 
$T$ above the critical temperature. The gas also has a certain entropy given by Eq. (6). We now ask ourselves what would happen if the system is cooled adiabatically to a lower temperature by slowly lowering the trapping frequency to a value $\omega^{\prime}$. Since during this adiabatic process the entropy and the number of particles in the gas remain constant, we know the answer to this question if we determine the final chemical potential and temperature combination $\left(\mu^{\prime}, T^{\prime}\right)$ such that $N\left(\mu^{\prime}, T^{\prime}, \omega^{\prime}\right)=N(\mu, T, \omega)$ and $S\left(\mu^{\prime}, T^{\prime}, \omega^{\prime}\right)=S(\mu, T, \omega)$.

The reason why we propose to adiabatically cool the gas in this manner is that for the ideal Bose gas, the only way to keep both the entropy as well as the particle number constant during the change $\omega \rightarrow \omega^{\prime}$ in the trapping frequency, is to change the temperature from $T$ to $\left(\omega^{\prime} / \omega\right) T$ and the chemical potential from $\mu$ to $\left(\omega^{\prime} / \omega\right) \mu$. This leaves all the occupation numbers of the harmonic oscillator states unchanged. More importantly, it leaves the fugacity and therefore the degeneracy parameter $n(\mathbf{0}) \Lambda_{t h}^{3}$ in the center of the trap unchanged. So for the ideal gas, adiabatic cooling does not bring us closer to criticality. Therefore, if we can reach BoseEinstein condensation in an interacting gas in this way, it must be entirely due to the effect of interactions.

In Fig. 2, the value of the degeneracy parameter in the center of the trap is plotted for five different adiabatic processes (solid lines 1 to 5) during an adiabatic ramp down of the trap frequency from a starting frequency of $373 \mathrm{~Hz}$. All five adiabatic curves correspond to a gas containing $N=20,000{ }^{87} \mathrm{Rb}$ particles with an $s$-wave scattering length of $109 a_{0}$.

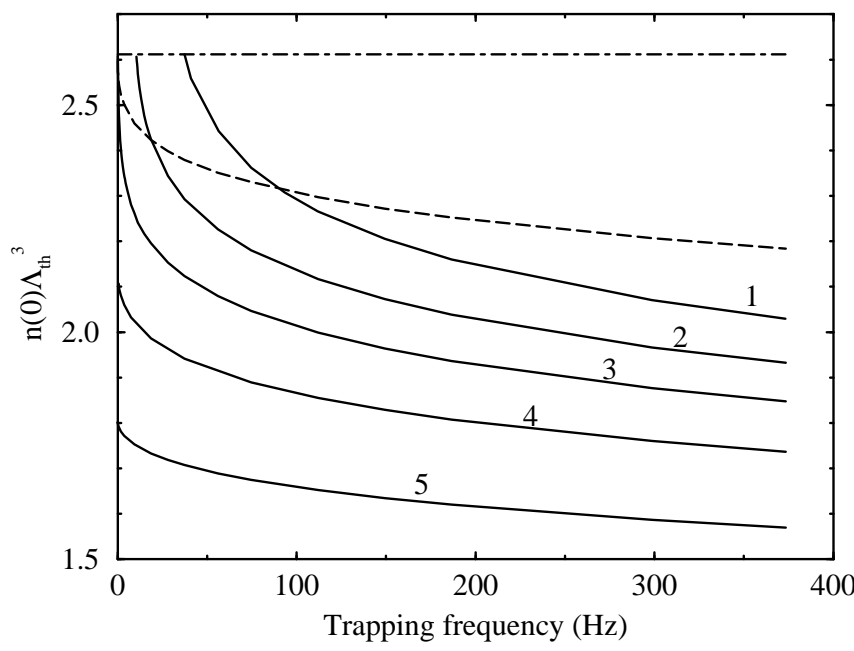

FIG. 2. Adiabatic trajectories (solid lines) of a gas of $20,000{ }^{87} \mathrm{Rb}$ particles when the trapping frequency is ramped down. The initial temperatures are 1) $442.21 \mathrm{nK}, 2) 445.85$ nK, 3) $449.43 \mathrm{nK}$, 4) $454.72 \mathrm{nK}$, and 5) $464.16 \mathrm{nK}$. The dashed line indicates the value for the central degeneracy parameter at which Bose-Einstein condensation first occurs if fluctuation effects are taken into account. The dot-dashed line denotes the mean-field critical degeneracy parameter.
The initial temperature of the gas increases from the upper to the lower curves and consequently the chemical potential increases from the lower to the upper curve, since the total number of particles is the same. Physically, for a nonideal gas the extra term $2 n(\mathbf{r}) T^{2 B} / k_{B} T$ in the exponent of $z(\mathbf{r})$ causes a deviation from a horizontal line, because the density does not scale linearly with $T$ but approximately with $T^{3 / 2}$. Thus keeping $N$ and $S$ constant when $\omega$ is varied, we now have to change the local fugacity and as a result the degeneracy parameter in the center of the trap also changes. Furthermore, to achieve Bose-Einstein condensation in a gas with a positive scattering length, it is clear that the frequency must be ramped down because this lowers the density of the gas and reduces the effect of the repulsive interaction $2 n(\mathbf{r}) T^{2 B}$. As a result, the density in the center of the trap reduces less than in the ideal gas case and the degeneracy parameter $n(\mathbf{0}) \Lambda_{t h}^{3}$ increases.

In Fig. 2 we also plotted the value of the critical degeneracy parameter following from the renormalization group theory of Ref. 12 and corresponding to the instantaneous temperature during the adiabatic cooling of the gas. It should be noted that in principle this curve is different for each adiabatic trajectory. However, for the five trajectories presented here the differences cannot be distinguished on this scale. Another aspect that can be seen from Fig. 2 is that only the upper three curves reach the conditions for Bose-Einstein condensation, whereas curves 4 and 5 remain uncondensed all the way down to $\omega=0$ which is equivalent to $T=0$. Indeed, a detailed analysis of the chemical potential and the central density as a function of the instantaneous temperature $T$ shows that for curves 1 to $3 \mu \propto T^{\alpha}$ and $n(\mathbf{0}) \propto T^{\beta}$ for the complete $\omega$-interval considered. Moreover, the powers obey $\alpha<\beta<3 / 2$ which implies that although initially the gas is not condensed and $\mu<2 n(\mathbf{0}) T^{2 B}$, there always will be some lower and nonzero temperature such that $\mu=2 n(\mathbf{0}) T^{2 B}$ and the gas fulfills the mean-field critical conditions. Consequently it will have already achieved the critical conditions including fluctuation effects at a higher temperature.

However, for curves 4 and 5 the chemical potential and central density exhibit a different behavior. This is due to the fact that during the cooling process the chemical potential becomes negative in order to satisfy conservation of both the number of particles $N$ as well as the entropy $S$. Therefore, the system cannot become Bose condensed unless $\mu \uparrow 0$ and $2 n(\mathbf{0}) T^{2 B} \downarrow 0$ for $T \rightarrow 0$. Looking again in more detail to the power behaviour of $\mu$ and $n(\mathbf{0})$ as a function of $T$, we find that $n(\mathbf{0}) \propto T^{\beta}$ with $\beta$ almost equal to $3 / 2$, and that $\mu \propto-T$ for $T \rightarrow 0$. So both $\mu$ and $2 n(\mathbf{0}) T^{2 B}$ indeed approach zero, but the limit of the ratio $\mu / k_{B} T$ will now decide whether or not the system achieves Bose-Einstein condensation at zero temperature and frequency.

Fig. 3 shows a plot of the trapping frequencies at which Bose-Einstein condensation first occurs if only mean-field effects are taken into account (curve 1) and if also fluctu- 
ation effects are included (curve 2). Again all adiabatic processes are for a gas containing 20,000 particles and an initial trapping frequency of $373 \mathrm{~Hz}$. The initial temperature of the gas is given by the ordinate. From this figure it is clear that the corrections due to fluctuations have a large influence on the final frequency at which Bose-Einstein condensation is achieved. For suitable initial conditions, the final trapping frequency can exceed the mean-field prediction by more than a factor of 3 . We therefore conclude that in principle the fluctuation corrections are experimentally observable by adiabatically cooling the gas.

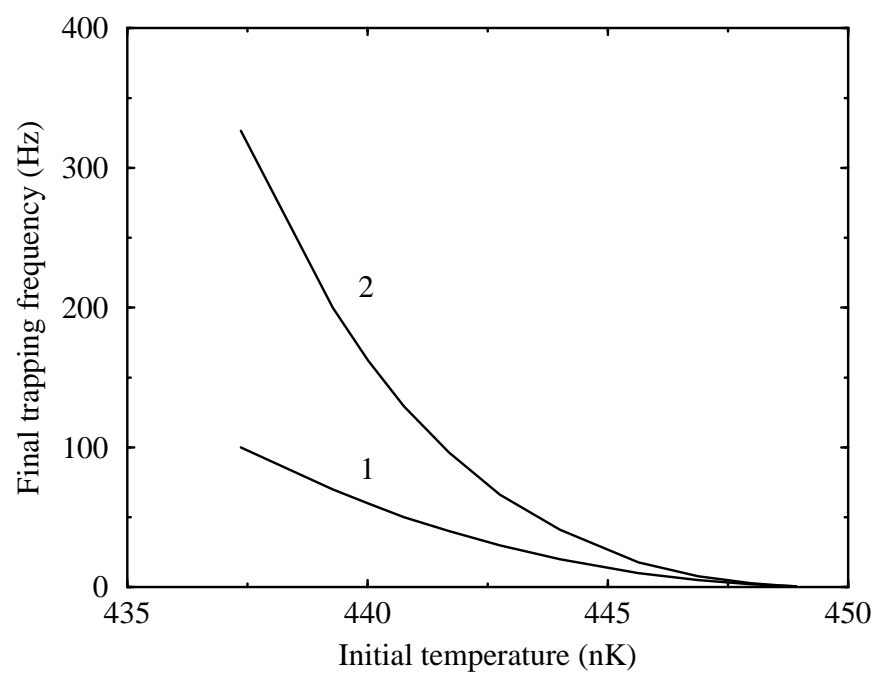

FIG. 3. Trapping frequency needed to obtain the critical conditions in the center of the trap, starting from a configuration of 20,000 particles in a $373 \mathrm{~Hz}$ trap and at a temperature denoted by the ordinate. Curve 1 is the mean-field result and curve 2 includes also the effect of fluctuations.

However, it must be noted that while curves 1 and 2 in Fig. 3 can be separated by as much as a factor of three in the frequency axis, they are separated by only $1 \%$ on the temperature axis. This should be compared with the JILA group's most recent attempt at absolute thermometry, with claimed absolute accuracy no better than $5 \%$ [6]. Nevertheless, measurements of the condensate fraction near the critical temperature indicate that the shot-to-shot reproducibility of the scaled temperature is much better, i.e. on the order of $1 \%$ [14]. Moreover, the scaled temperature varies smoothly with final evaporative rf cut, which is a readily adjustable experimental parameter. We therefore envision an experiment in which samples are prepared at temperatures precisely determined relative to the empirical onset temperature of BEC, and then subjected to adiabatic ramps of the trapping frequency.

In summary, we showed that, although fluctuation corrections decrease the critical density of a trapped gas of repulsively interacting bosonic atoms, they do not com- pletely cancel the increase in the critical number of particles obtained from mean-field theory. The effect of the interactions, and in particular, of the fluctuation corrections to the mean-field theory can be observed more directly by adiabatically cooling the gas to criticality. Here we imply changing adiabatically the frequency of a harmonic trap and not the power law of the trapping potential, which has recently been shown to change already the degeneracy parameter of the ideal Bose gas [15]. In addition to being more difficult to implement experimentally, the latter approach is not so favorable for our purposes because it is less sensitive to the effect of interactions. We hope that this work might stimulate an experimental observation of these interesting phenomena in the near future.

We acknowledge stimulating conversations with Michel Bijlsma, Jason Ensher, Deborah Jin, and Sandro Stringari. E.C. acknowledges support from the O.N.R. and the N.S.F.

[1] M.H. Anderson, J.R. Ensher, M.R. Matthews, C.E. Wieman, and E.A. Cornell, Science 269, 198 (1995).

[2] K.B. Davis, M.O. Mewes, M.R. Andrews, N.J. van Druten, D.S. Durfee, D.M. Kurn and W. Ketterle, Phys. Rev. Lett. 75, 3969 (1995).

[3] C.C. Bradley, C.A. Sackett, J.J. Tollett and R.G. Hulet, Phys. Rev. Lett. 75, 1687 (1995); C.C. Bradley, C.A. Sackett, and R.G. Hulet, Phys. Rev. Lett. (to be published).

[4] D.S. Jin, J.R. Ensher, M.R. Matthews, C.E. Wiemann, and E.A. Cornell, Phys. Rev. Lett. 77, 420 (1996).

[5] M.-O.Mewes, M.R. Andrews, N.J. van Druten, D.M. Kurn, D.S. Durfee, C.G. Townsend, and W. Ketterle, Phys. Rev. Lett. 77, 988 (1996).

[6] J.R. Ensher, D.S. Jin, M.R. Matthews, C.E. Wieman, and E.A. Cornell, Phys. Rev. Lett. (to be published).

[7] A.L. Fetter, Phys. Rev. A 53, 4245 (1996).

[8] S. Stringari, Phys. Rev. Lett. 77, 2360 (1996).

[9] K.G. Singh and D.S. Rokhsar, Phys. Rev. Lett. 77, 1667 (1996).

[10] M. Edwards, P.A. Ruprecht, K. Burnett, R.J. Dodd, and C.W. Clarck, Phys. Rev. Lett. 77, 1671 (1996).

[11] S. Giorgini, L.P. Pitaevskii and S. Stringari, Phys. Rev. A (to be published).

[12] M. Bijlsma and H.T.C. Stoof, Czech. J. Phys. 46, 553 (1996) and Phys. Rev. A 54, 5085 (1996).

[13] M. Houbiers and H.T.C. Stoof, Phys. Rev. A 54, 5055 (1996).

[14] D.S. Jin (private communication).

[15] P.W.H. Pinske, A. Mosk, M. Weidemüller, M.W. Reynolds, T.W. Hijmans, and J.T.W. Walraven (unpublished). 